\title{
Radiological diagnosis of recurrent colonic carcinoma at the anastomosis
}

\author{
CLIVE BARTRAM AND JOHN E. HALE
}

From the Westminster Hospital, London

SUMMARY The barium enemas of 23 cases of end-to-end anastomosis following resection of a colonic carcinoma demonstrated that the normal anastomosis should be smooth, symmetrical, and have a regular mucosal pattern. In contrast the three patients reported with proven recurrent carcinoma at the anastomosis showed irregular asymmetrical filling defects ${ }^{\mathbf{1}}$.

As a base line the anastomosis should be investigated with a limited double-contrast enema examination six weeks after operation. Earlier examinations may show irregularity due to incomplete healing.

Following resection of a colonic or rectal carcinoma further symptoms of abdominal pain and rectal bleeding may be due to recurrent tumour at the anastomosis. If this is beyond reach of the sigmoidoscope a barium enema will be required, but interpretation of the radiological finding may be difficult, especially in the early postoperative period when some degree of constriction at the anastomosis is common. Three patients who developed an anastomotic recurrence are reported. In two of these cases the significance of the asymmetrical anastomosis seen on barium enema was minimized and interpreted as being caused by the particular type of suturing. In order to establish what can be accepted as a normal radiological appearance of a colonic anastomosis, 23 barium enemas given after operation to patients with excised colonic carcinoma were studied.

\section{Case 1}

One year after resection of a sigmoid carcinoma

Received for publication 13 February 1970.

'A paper was read to the proctological section of the Royal Society of Medicine entitled 'Four cases with a tumour recurrence at the colonic anastomosis' on 28 January 1970 by J.E.H. and two of those cases are included in the present series. a routine barium enema revealed an asymmetrical anastomosis and mucosal irregularity in a man aged 44. This was attributed to inversion of the bowel by sutures (Fig. 1). Because of increasing abdominal pain, a repeat barium enema was performed some months later to reveal a large mass at the anastomosis (Fig. 2). The recurrence was excised, and examination of the specimen confirmed that it was histologically similar to the original tumour and arose in the anastomosis.

\section{Case 2}

Two months following resection of a small annular sigmoid carcinoma, the patient, a woman of 68 , experienced increasing constipation. The anastomosis seen on barium enema displayed an asymmetrical filling defect which was thought to be compatible with the appearances one might expect two months after operation (Fig. 3). Symptoms increased, and five months after operation a further barium enema showed a filling defect at the anastomosis (Fig. 4). Laparotomy confirmed the diagnosis, and a tumour at the anastomosis was excised. In the specimen (Fig. 5) a suture can be seen surrounded by recurrent carcinoma. 


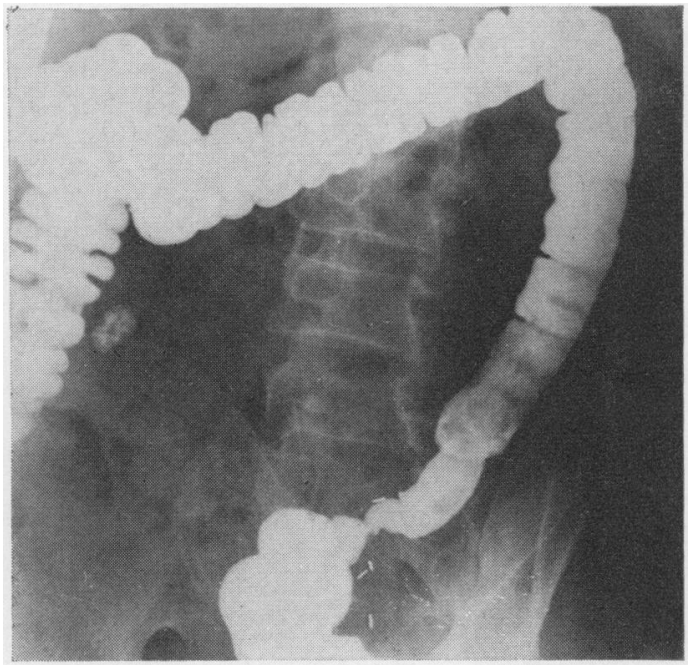

Fig. 1 Case 1: irregularity at anastomosis one year after resection.

\section{Case 3}

One year following resection of a sigmoid carcinoma in a 50-year-old man, rectal bleeding occurred and a repeat barium enema (Fig. 6) displayed an irregular filling defect at the anastomosis. A diagnosis of recurrent tumour of the anastomosis was made, and at operation a small carcinoma arising in the suture line was widely excised.

\section{Barium Enemas after Resection of Colonic Carcinoma}

In order to differentiate radiologically a local recurrence from the constriction which may occur after colonic anastomosis, appreciation of the normal appearances of barium enemas after operation is required. The paucity of references to this problem prompted us to review a series of postoperative barium enemas in patients with excised colonic tumour. In the 23 cases studied the interval between operation and radiological examination varied from a few weeks to more than six years, although the great majority of the enemas were performed within the first year after operation. A standard barium enema technique has been employed and most measurements were taken from the filled over-couch film. Two patients also had a limited doublecontrast barium enema. The surgical technique was similar in all patients, and consisted of a conventional two-layer, end-to-end anastomosis using continuous catgut and interrupted thread sutures. In all but three of the 23 patients a

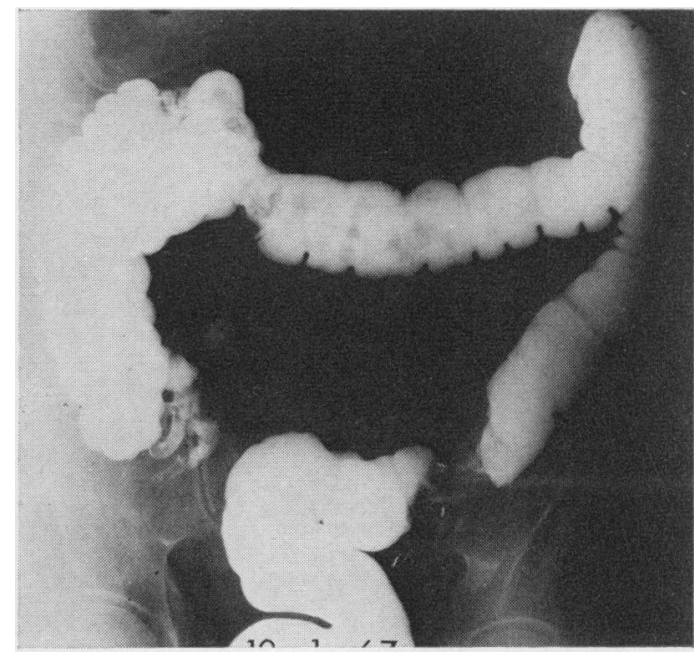

Fig. 2 Repeat enema in case 1 showing an increasing mass at anastomosis.

constant constriction could be seen at the site of anastomosis, the contour of which was smooth and symmetrical (Fig. 7). The double contrast films showed normal mucosal folds crossing the anastomosis. The length of constriction varied from $0.3 \mathrm{~cm}$ to $2.5 \mathrm{~cm}$, but the majority were under $1 \mathrm{~cm}$. The calibre of the anastomosis measured at its narrowest point was between $0.4 \mathrm{~cm}$ and $2.5 \mathrm{~cm}$ (average $1.5 \mathrm{~cm}$ ) and the ratio of this to the adjacent normal distal bowel was $2: 7$ on average.

In two patients, who had more than one enema after operation, subsequent films showed no change in the constriction which, in one case, could still be seen six years after operation.

\section{Discussion}

It has been estimated that at the anastomosis $50 \%$ of the recurrences following resection of tumours of the large bowel and rectum result from reimplantation of malignant cells, whilst the remainder are due to inadequate excision. (Goligher, Dukes, and Bussey, 1951). Since the introduction of a technique of colonic irrigation with some cancerocidal solution before anastomosis the incidence of suture line recurrences due to implantation has been reduced to under $2 \%$ (Keynes, 1961).

If the anastomosis is beyond reach of a sigmoidoscope, early diagnosis of an anastomotic recurrence will depend on the correct interpretation of a barium enema. The radiological appearances in the three patients who were subsequently found to have a recurrence all 


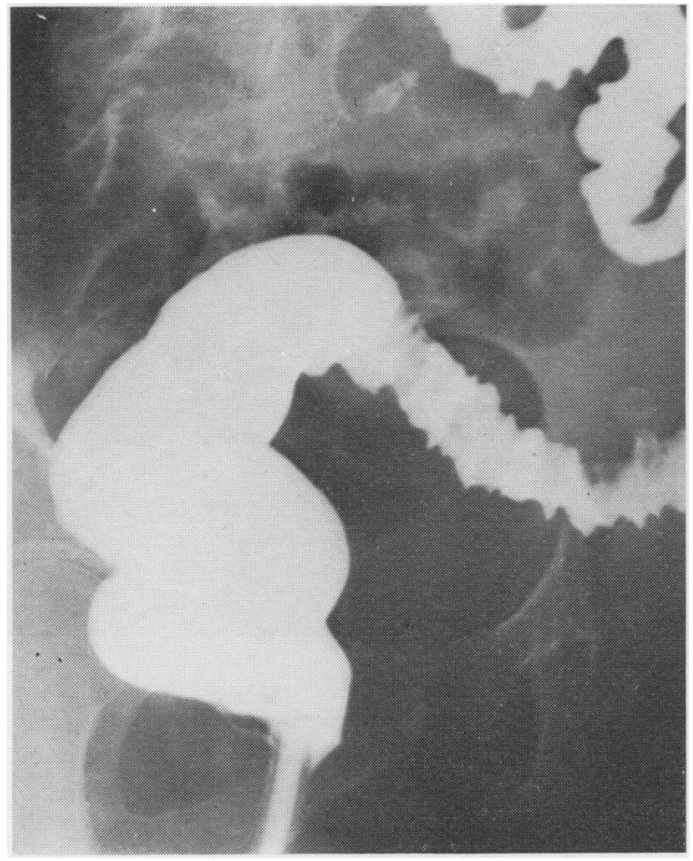

Fig. 3 Case 2: two months after resection, the filling defect at the inferior margin of the anastomosis is thought to be due to incomplete healing.

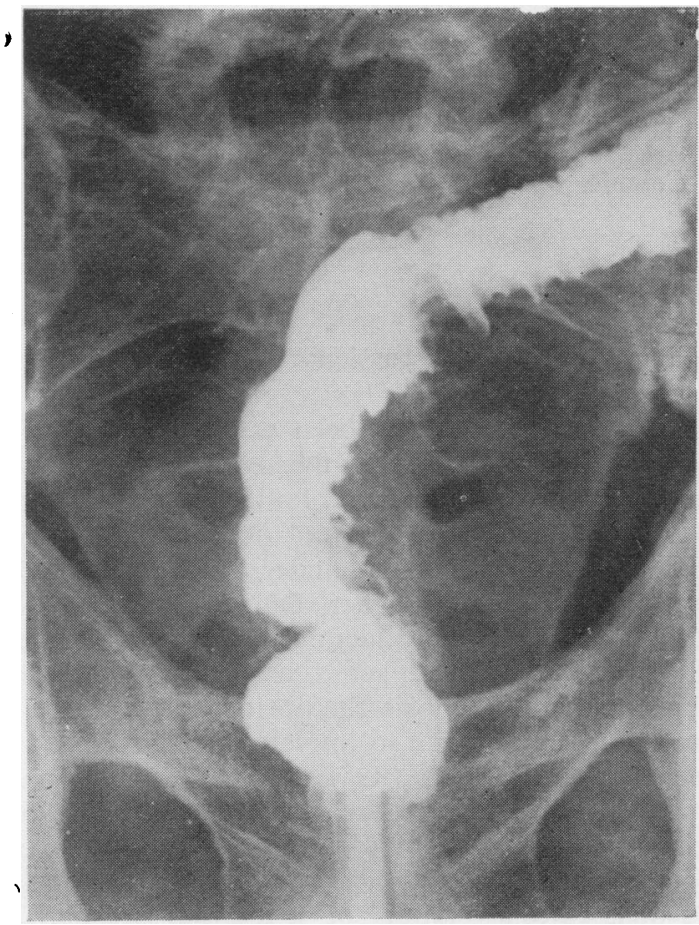

Fig. 4 Case 2: five months after resection the irregularity appears larger.

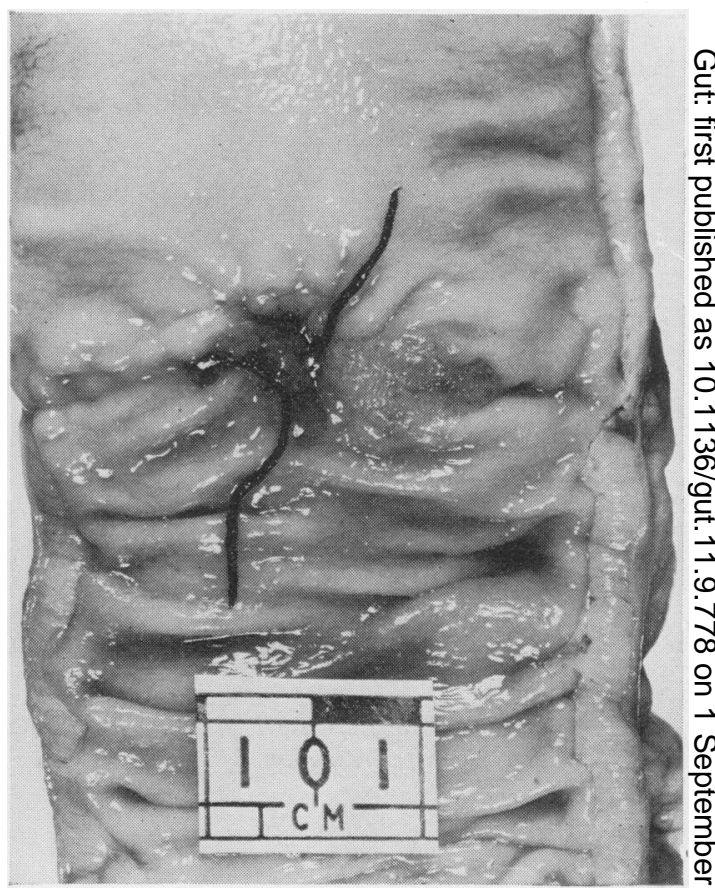

Fig. 5 Case 2: resected specimen shiowing tumour at the anastomosis with embedded suture.

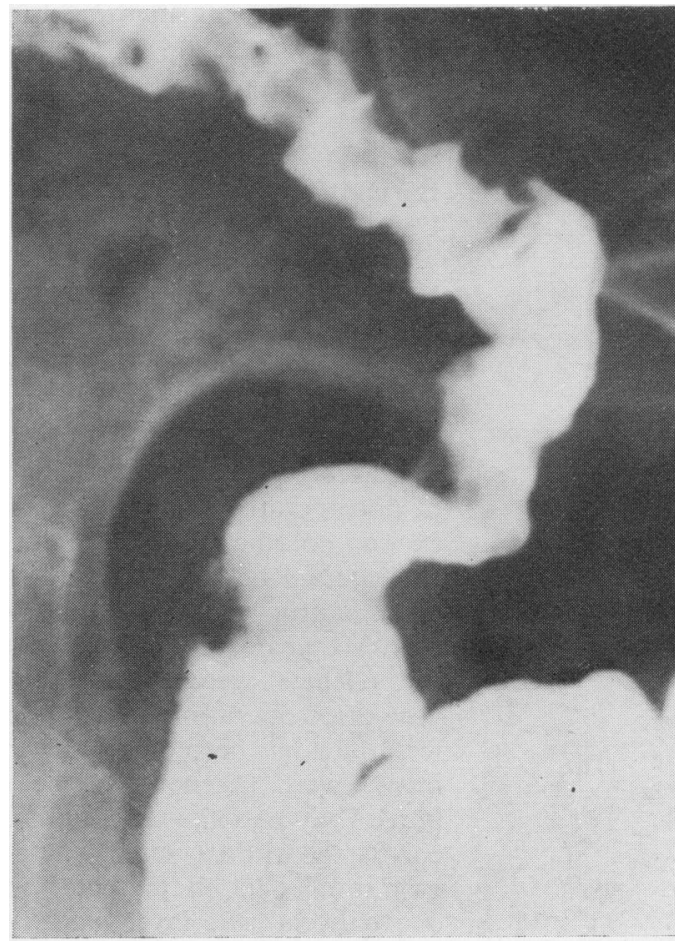

Fig. 6 Case 3: one year after resection, showing the irregular filling defect at the anastomosis. 


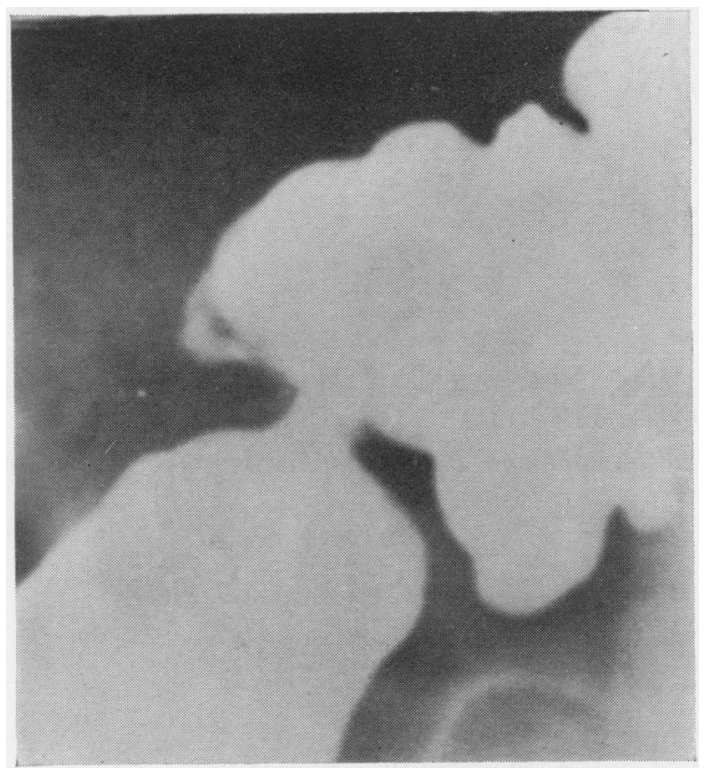

Fig. 7 Normal anastomosis showing smooth and symmetrical walls.

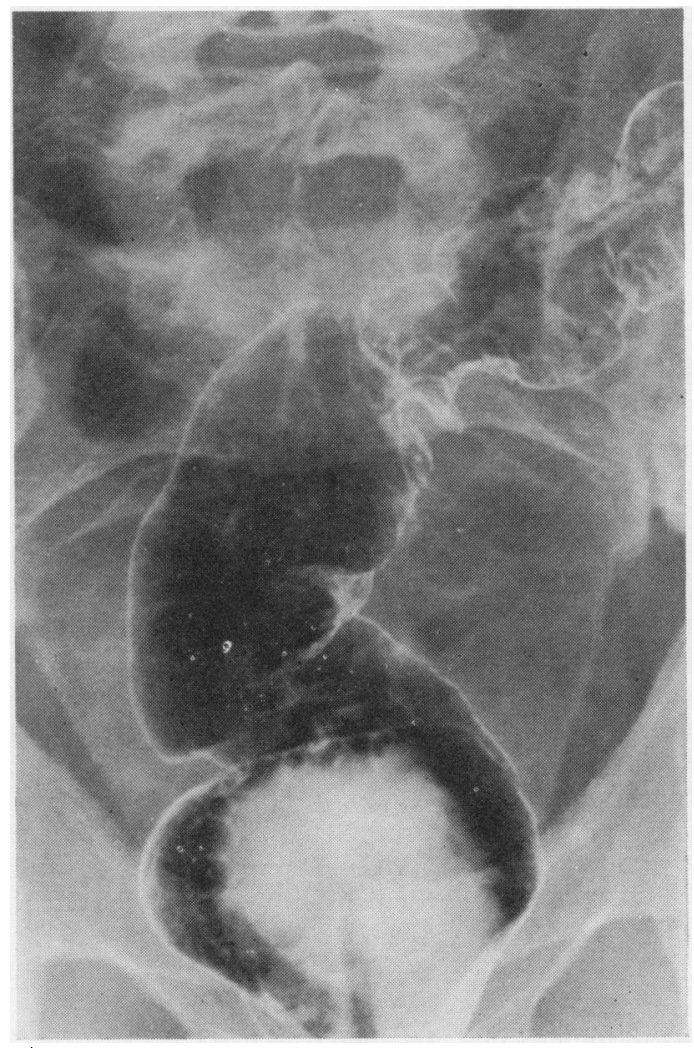

Fig. 8 Double-contrast enema showing irregular mass and loss of mucosal folds due to recurrent carcinoma. showed an asymmetrical filling defect at the anastomosis. This was in direct contrast to the barium enema films of the 23 other patients reviewed. In these cases constriction at the anastomosis was symmetrical and smooth-walled with a normal mucosal pattern. Our findings are supported by Sharpe and Golden (1950) who studied the radiological appearances of 42 cases of colonic anastomosis at various intervals after operation. Only three showed no abnormality at the anastomotic site, and in the other 39 cases a constriction usually less than $1 \mathrm{~cm}$ was demonstrated. The constriction was symmetrical in all but four patients, and, of these, three were found to have recurrent carcinoma a were anastomosis. In the remaining case adhesionst the responsible for the unilateral filling defect.

The interval between operation and barium enema is an important factor affecting the radiological appearance. In two of our patients initial barium enemas performed three weeks after operation showed gross mucosal irregularity and constriction, whereas subsequent barium enemas revealed a normal symmetrical constriction. Fleischner and Berenberg (1956) emphasized that the barium enema soon after operation may show some temporary irregularity of the anastomosis due to oedema and spasm. Healing normally takes about six weeks, by which time the configuration of the anastomosis should have become permanent. We suggest that, as a base line, a barium enema should be performed some six weeks after operation. A double-contrast enema is of additional help in demonstrating the mucosal pattern (Fig. 8). A limited examination can be performed by allowing barium to run in just past the anastomosis. Air is then blown into the colon to distend the anastomosis and spot films of it can be taken. If there is any suspicion of a mucosal irregularity or a filling defect at the anastomosis, then a further limited barium enema one month later with careful scrutiny of the anastomosis is essential. By this time either healing will be completed and the anastomosis appear normal or there will be definite evidence of a recurrence.

We are grateful to Professor Harold Ellis and Dr J. Gleeson for their encouragement in preparing this paper.

References

Fleischner, F. G., and Berenberg, A. L. (1966). Recurrent carcinoma of the colon at the site of the anastomosis. Radio$\log y, 66,540-547$.

Goligher, J. C., Dukes, C. E., and Bussey, H. J. R. (1951) Local recurrences after sphincter-saving excisions for carcinoma of the rectum and recto-sigmoid. Brit. $J$. Surg., 39, 199-211.

Keynes, W. M. (1961). Implantation from the bowel lumen in cancer of the large intestine. Ann. Surg., 153, 357-364.

Sharpe, M., and Golden, R. (1950). End-to-end anastomosis of the colon following resection. A Roentgen study of forty-two cases. Amer. J. Roentgenol., 64, 769-777. 